\title{
A WALL-MODELED/WALL-RESOLVED LES METHOD FOR TURBULENT WALL FLOWS
}

\author{
Francesco De Vanna ${ }^{1}$, Michele Cogo ${ }^{1}$, Matteo Bernardini ${ }^{2}$, Francesco Picano ${ }^{1}$ and \\ Ernesto Benini ${ }^{1}$ \\ ${ }^{1}$ Department of Industrial Engineering, Universitá di Padova, Via Venezia 1, 35131 Padova, Italy, \\ francesco.devanna@unipd.it \\ ${ }^{2}$ Department of Mechanical and Aeronautical Engineering, Universitá di Roma 'La Sapienza", Rome, \\ Italy
}

Key words: Large-Eddy Simulation, Wall-modeling, Turbulence, Wall Flows

\begin{abstract}
This present paper reports a novel methodology to simulate wall-bounded flows in the LargeEddy Simulation framework using an automatic transition between a wall-modelled and a wall-resolved approach. The proposed technique aims at prescribing the right (modelled or resolved) wall shear stress and wall heat flux, preserving at the same time the no-slip/no-penetration conditions for the velocity and the isothermal/adiabatic conditions for the temperature fields. The approach is successfully implemented in a high-order finite-difference framework, and it is found able to adapt smoothly to the available nearwall grid spacing. Thus, the method falls into the wall-resolved case, when the near-wall dynamic is directly computed, whereas it employs the wall stress model when a full resolution of the near-wall region is not achievable. The method is tested on a nearly-incompressible turbulent channel flow and a supersonic spatially-devolving boundary layer flow. The obtained results highlight an excellent accuracy in representing the wall turbulence dynamics in terms of mean velocity profiles and fluctuations, almost independently of the near-wall spatial resolution. Thus, the proposed method results in a promising technique for analysis of high-Reynolds wall-bounded flows.
\end{abstract}

\section{INTRODUCTION}

Modelling wall turbulence is a crucial task in a broad range of engineering applications, such as external aerodynamics, turbomachinery, propulsive and hydrodynamics systems [1, 2, 3, 4]. In numerical simulations of turbulent flows, the most commonly used scale-resolving approach in the academic world is Large-Eddy Simulation (LES). LES has the main benefit that the dynamics of the energy-dominant and flow-dependent large eddies are directly resolved rather than being modelled, as they would be with a standard Reynolds-Averaged Navier-Stokes (RANS) approach. However, the presence of solidboundaries represents the major challenge in terms of computational cost since, in these regions, the scales of motion are of fundamental importance for the overall system dynamics, which means that the LES grid must resolve them. At the same time, the wall-eddies are orders of magnitude smaller than the outside boundary layer's scales. As a result, most of the computational effort in wall-flows is dedicated to the near-wall regions, leading to prohibitive calculation turn around times for a Wall-Resolved LES (WRLES). 
Chapman [5] and Choi and Moin [6] estimated that the number of grid points necessary to resolve the near-wall eddies for a Direct Numerical Simulation (DNS) and Wall-Resolved LES (WRLES) is about $N_{D N S} \sim R e_{L}^{37 / 14}$ and $N_{W R} \sim R e_{L}^{13 / 7}$, respectively, where $R e_{L}$ is the Reynolds number based on a characteristic length. It is easy to see that both DNS and WRLES becomes unpractical at high Reynolds numbers; thus, a strategy where only the outer-layer eddies are resolved and the near-wall eddies are modelled is required [7]. The proposed method falls in the Wall-Modelled LES (WMLES) path and deals with a wall-stress model to account for the near-wall dynamics. In particular, the process aims at feeding the information of the wall shear stress, $\tau_{w}$, and the wall heat flux, $q_{w}$, computed with an external model into the LES field. Recent developments about WMLES are reported in the work of Larsson et al. [8], Bose and Park [9] and Yang and Lv [10].

The primary purpose of this work is to provide an automatic transition between the WRLES and WMLES techniques, which are still separate strategies in the field of wall-turbulence applications. Considering, for example, a high Reynolds number flow over an airfoil, the most computational demanding regions (i.e., turbulent zones) can be accurately represented by a wall model to save resources. In contrast, regions characterised by incipient separation, in which the velocity gradient is locally zero, might be directly computed on the grid. Thus, the unification between WMLES and WRLES techniques could be desirable in always relying on the best possible wall resolution while keeping the overall computational cost acceptable. In this sense, the proposed approach can be considered a unified WR/WMLES method.

The present paper is organised as follows: in Section 2, the governing equations and the numerical method are presented and discussed. Section 3 provides the results discussion. Finally, Section 4 sum-

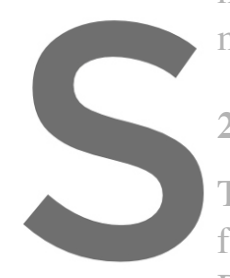
marises the conclusion

2 GOVERNING EQUATIONS

The present study is carried out $v$ fully compressible Navier-Stoke
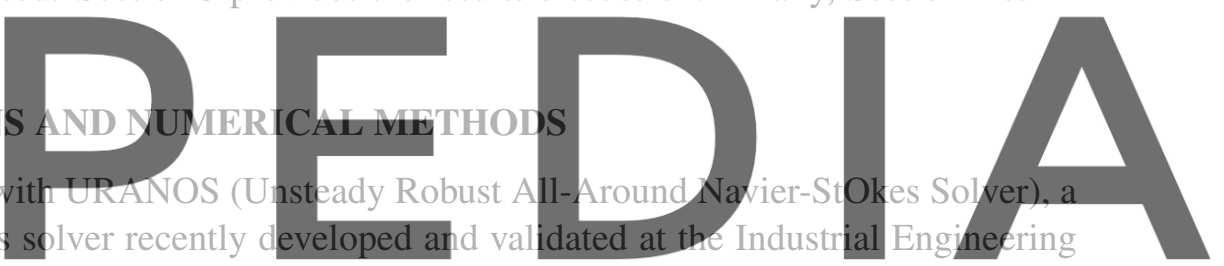

Department of the University of Padua $[11,12,13]$. The solver implements the Favre-filtered Navier-

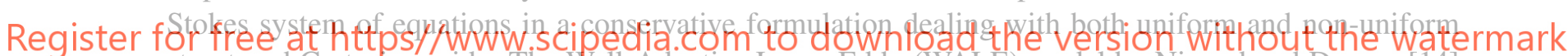
structured Carteslan grids. The Wall-Adaptive Large-Eddy (WALE) model by Nicoud and Ducros [14] is employed accounting for the SubGrid-Scale (SGS) contributions. The model provides the correct $\bar{\mu}_{S G S} / \bar{\mu} \sim O\left(y^{+}\right)^{3}$ asymptotic scaling in the near-wall regions without prescribing any artificial transition between the bulk flow and the boundary layer. Furthermore, in the present unified WR/WMLES implementation, the model guarantees the proper transition between a Wall-Modelled (WM) and a WallResolved (WR) configuration since the SGS viscosity automatically vanishes in the near-wall regions. A High-Order Finite-Difference (HOFD) framework is adopted, employing a $5 t h$-order accurate hybridcentral WENO scheme to reconstruct the convective terms. The 6 th-order conservative central-finitedifference scheme by De Vanna et al. [15] is employed for diffusive contributions. The solution is advanced in time with a $3 r d$-order low-storage Total Variation Diminishing (TVD) Runge-Kutta scheme. The scheme provides a maximum Courant-Friedrich-Lewy parameter $C F L_{\max }$ equal to 1 . In the authors' computations, the $C F L$ number is set equal to 0.5 , a value which represents a good compromise between stability and speed of the calculations. The wall-stress-model is based on the set of ordinary differential equations proposed by Larsson et al. [8], that consists in the simplified momentum and total energy equations assuming the equilibrium assumption (for the details the reader is referred to $[8,16]$ ). 
These equations need to be solved in an independent grid that goes from the wall $y=0$ (where the no-slip/no-penetration conditions for velocity and the isothermal/adiabatic wall conditions for temperature are imposed), to a matching location $y=h_{\text {wall }}$ where the model takes as boundary conditions the quantities sampled by the LES field. The ODE system solution returns the near-wall velocity $U_{w m}$ and temperature fields $T_{w m}$, providing the computation of the wall shear stress, $\tau_{w, w m}$ and the wall heat flux $q_{w, w m}$, defined as

$$
\tau_{w, w m}=\left(\mu \frac{d U_{w m}}{d y}\right)_{y=0}, \quad q_{w, w m}=\left(\frac{c_{p} \mu}{\operatorname{Pr}} \frac{d T_{w m}}{d y}\right)_{y=0}
$$

These values are fed as boundary conditions to the LES field. In particular, the proposed numerical strategy aims at modifying the wall SGS viscosity $\mu_{w}^{S G S}$ and wall SGS diffusivity $\lambda_{w}^{S G S}$ accounting for the proper wall shear-stress and wall heat-flux. Thus, $\mu_{w}^{S G S}$ and $\lambda_{w}^{S G S}$ are locally modified in such a way that a factor corrects the resolved velocity and temperature gradients providing the right $\tau_{w, w m}$ and $q_{w, w m}$ at the bound. This is accomplished through the effective wall viscosity and effective wall heat diffusivity, defined as

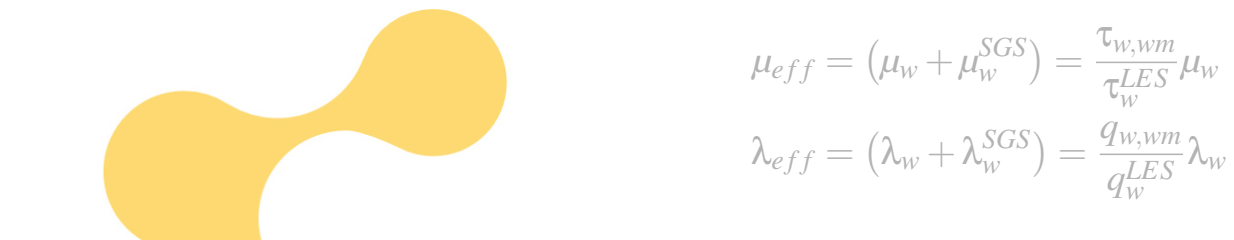

The information of $\mu_{e f f}$ and $\lambda_{e f f}$ is used to modify the SGS viscosity and diffusivity in such a way that the LES computation s values at the boundary. with the LES solver, accor resolution. Therefore, location are not zero but. WR case. This arranger

both the no-slip/no-penetration conditions for the velocity and the isothermal/adiabatic conditions for the Register fomfreeduat frattps/tha/waw.scipedia.com to download the version without the watermark

In the present method, great attention is paid to the matching location between the wall-stress model and the LES fieid. In particular, this is usuaily chosen as a fixed position, at a certain distance from the wall (see, e.g., Larsson et al. [8] for details), a choice which clashes with the dynamism of the proposed strategy and with the possibility of treating portions of the same wall with a WR or a WM approach according to local flow conditions. Thus, the matching location between the wall-stress model and the LES field is obtained using a dynamical procedure that checks if the first grid point near the wall $\left(y_{1}\right)$ satisfies the WRLES grid requirements, which are assumed by the condition $y_{w}^{+}=\rho_{w} u_{\tau} y_{1} / \mu_{w}<5$. In particular, a first $y_{w}^{+}$guess is obtained by solving the Reichardt's law [17] in term of the friction velocity $u_{\tau}$. The equation reads as

$$
\frac{u_{/ /}}{u_{\tau}}=\kappa^{-1} \log \left(1+\kappa y^{+}\right)+C_{\kappa}\left(1-e^{-\frac{y^{+}}{11}}-y^{+} \frac{e^{-0.33 y^{+}}}{11}\right)
$$

Here $\kappa=0.41$ is the Von Kármán constant while $C_{\mathrm{\kappa}}=7.8$ is the model fitting parameter. The information of $u_{\tau}$ is used to test the boundary resolution. In particular, if $y_{w}^{+}<5$, the local bound is treated as a WR; 
otherwise, the WM procedure is employed. The exchange location between the model and the LES field is always chosen to lie well within the $\log$-layer $\left(y_{h_{w m}}^{+} \approx 40\right)$ to avoid the extreme velocities and pressure fluctuations that characterise the buffer layer and to stabilise the response of the wall-stress model. A sketch related to the prominent near-wall occurrences is reported Figure 1.

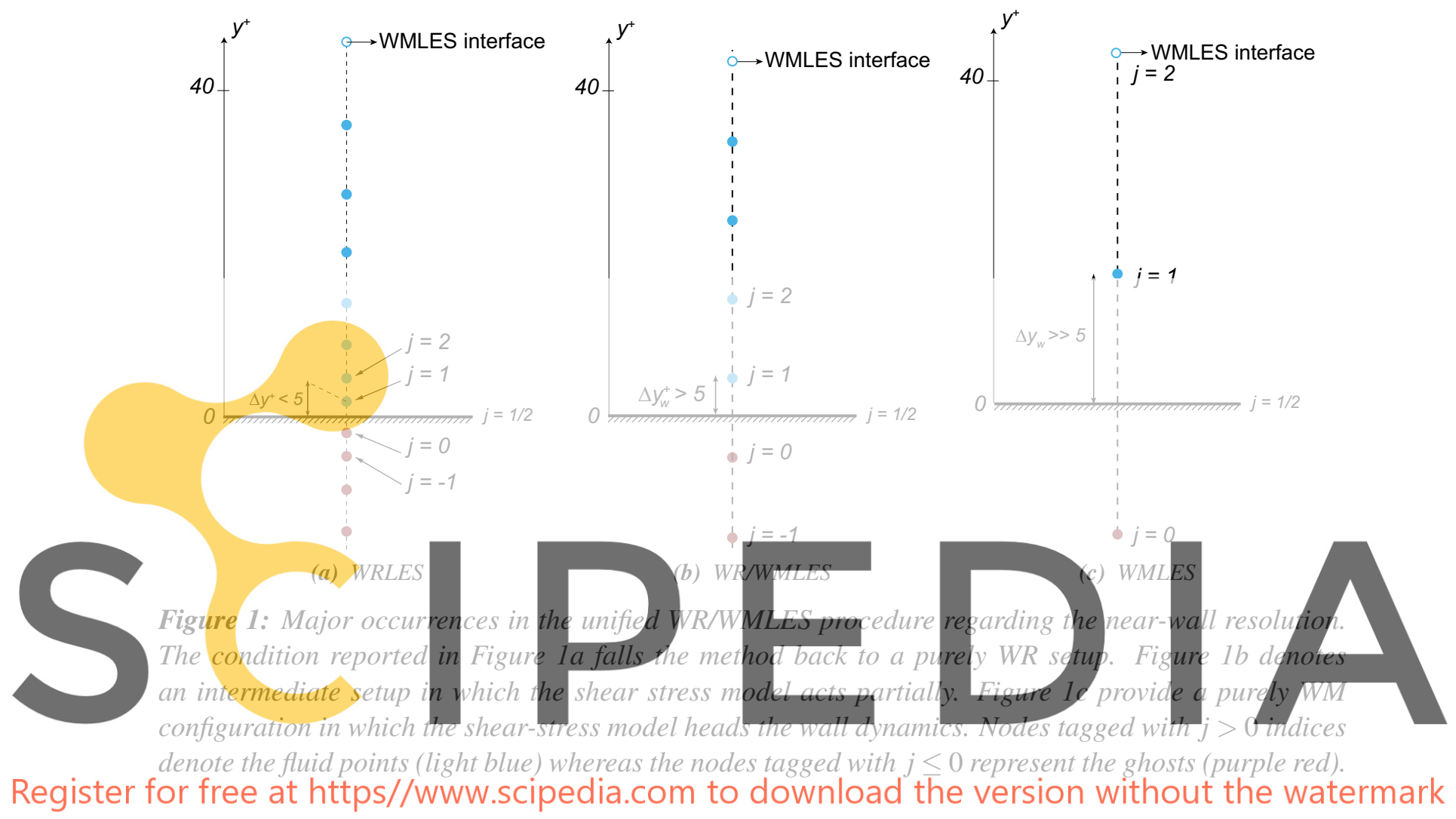

After the local near-wall resolution is guessed, and the matching location is found, the coupled equations are solved using a second-order accurate interpolation and differentiation at the cell faces, using the interface location $h_{w m}$ in the LES domain as a top boundary for the ODE model (see, e.g., $[16,8]$ for a detailed discussion). Since both the discretised equations result in a tridiagonal system, a classical TriDiagonal Matrix Algorithm (TDMA) is used to solve them efficiently. The desired effective viscosity $\mu_{e f f}$ (diffusivity $\lambda_{e f f}$ ) is enforced at the wall by means of the ghost nodes, in which are placed the ghost values of the overall viscosity $\mu_{t o t}=\mu+\mu_{S G S}$ (diffusivity $\lambda_{t o t}=\lambda+\lambda_{S G S}$ ). Thus, using the following extrapolation formula:

$$
\mu_{-j+1, g h}^{t o t}=2 \mu_{e f f}-\mu_{j, i n}^{t o t} \quad j=1, \ldots, 3
$$

This method is found to be very effective in terms of stability since it does not directly alter the LES velocity and temperature fields, but it locally modifies the quantities provided by the SGS model. 


\section{RESULTS DISCUSSION}

In this section, the main results obtained with the proposed unified WR/WMLES method are presented. Two distinct flow configurations are considered: a nearly-incompressible turbulent channel flow and a spatially-developing supersonic turbulent boundary layer.

\subsection{Turbulent channel flow}

The turbulent channel flow represents a fundamental benchmark for any wall-turbulence model. Here a nearly-incompressible flow at $M_{b}=u_{b} / c_{w}=0.1$ and $R e_{\tau}=\rho_{w} u_{\tau} h / \mu_{w}=590$ is considered. The details on the computational set-up and boundary conditions can be found looking at Bernardini et al. [18]. Since the main focus of the present work is to investigate the possibility of enabling a smooth transition between a WR and WM case, numerical simulations are performed using a set of grids with a decreasing wall-normal resolution. In particular, the pre-computed $y_{w}^{+}$location of the first grid point is progressively moved away from the wall in the following fashion: $y_{w}^{+}=\{3,19\}^{T}$. These values are selected to represent two possible conditions: nearly WR and mixed WR/WM arrangements. Figure 2 reports the results of the computations in terms of scaled mean velocity profile $u^{+}=u / u_{\tau}$ and density scaled Reynolds stresses components $\tau_{i j} / \tau_{w}$ and compares them with the DNS data of Vreman and Kuerten [19].
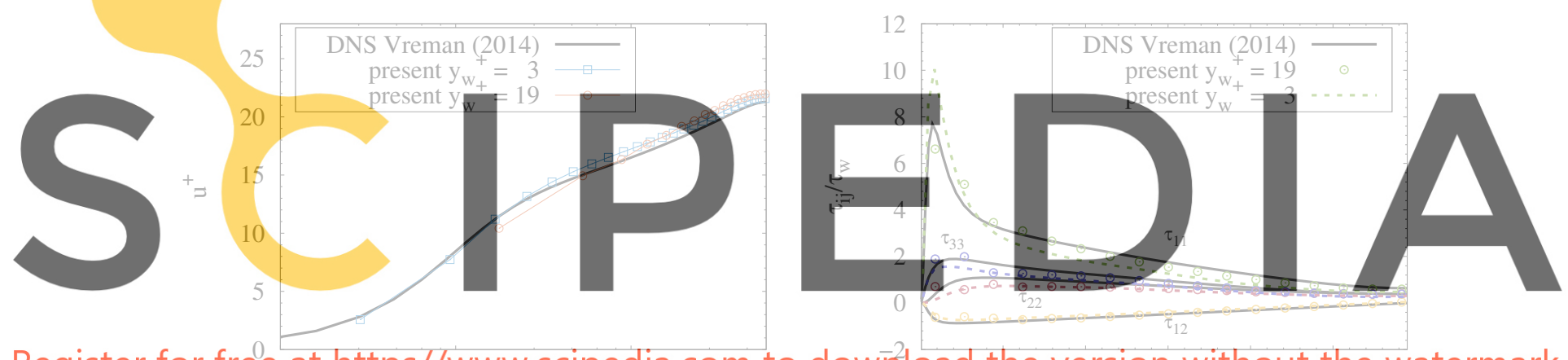

Register for free ${ }_{1}$ at https//www.scipedia.com to dowñload the versiobowithout 故e watermark

(a) Velocity profile

(b) Reynolds s stress components

Figure 2: Turbulent channel flow with a progressively grid derefinement. Figure $2 a$ and Figure $2 b$ show the scaled velocity profiles $u^{+}=\tilde{u} / u_{\tau}$ and the scaled Reynolds stress components $\bar{\rho} u_{i}^{\prime \prime} u_{j}^{\prime \prime} / \tau_{w}$, respectively. The results are computed using progressively coarser grids with a first internal location of $y_{w}^{+}=\{3,19\}^{T}$. Present data are compared with the incompressible DNS of Vreman and Kuerten [19] at Ma $a_{b}=0.1$ and $R e_{\tau}=590$.

The results highlight that the proposed method can link the WR and the WM successfully, even in the case where the first point falls inside the buffer layer. To quantitatively analyse the transition between the WR and the WM case, the two-points spanwise correlation of the turbulent streamwise velocity 
fluctuations is computed as

$$
R_{11}(y, \Delta z)=\frac{\widehat{u_{1}^{\prime \prime}(x, y, z) u_{1}^{\prime \prime}(x, y, z+\Delta z)}}{\widehat{u_{1}^{\prime \prime}(x, y, z) u_{1}^{\prime \prime}(x, y, z)}}
$$

Contours of $R_{11}$ as a function of the spacing $\Delta z$ are reported in Figure 3.
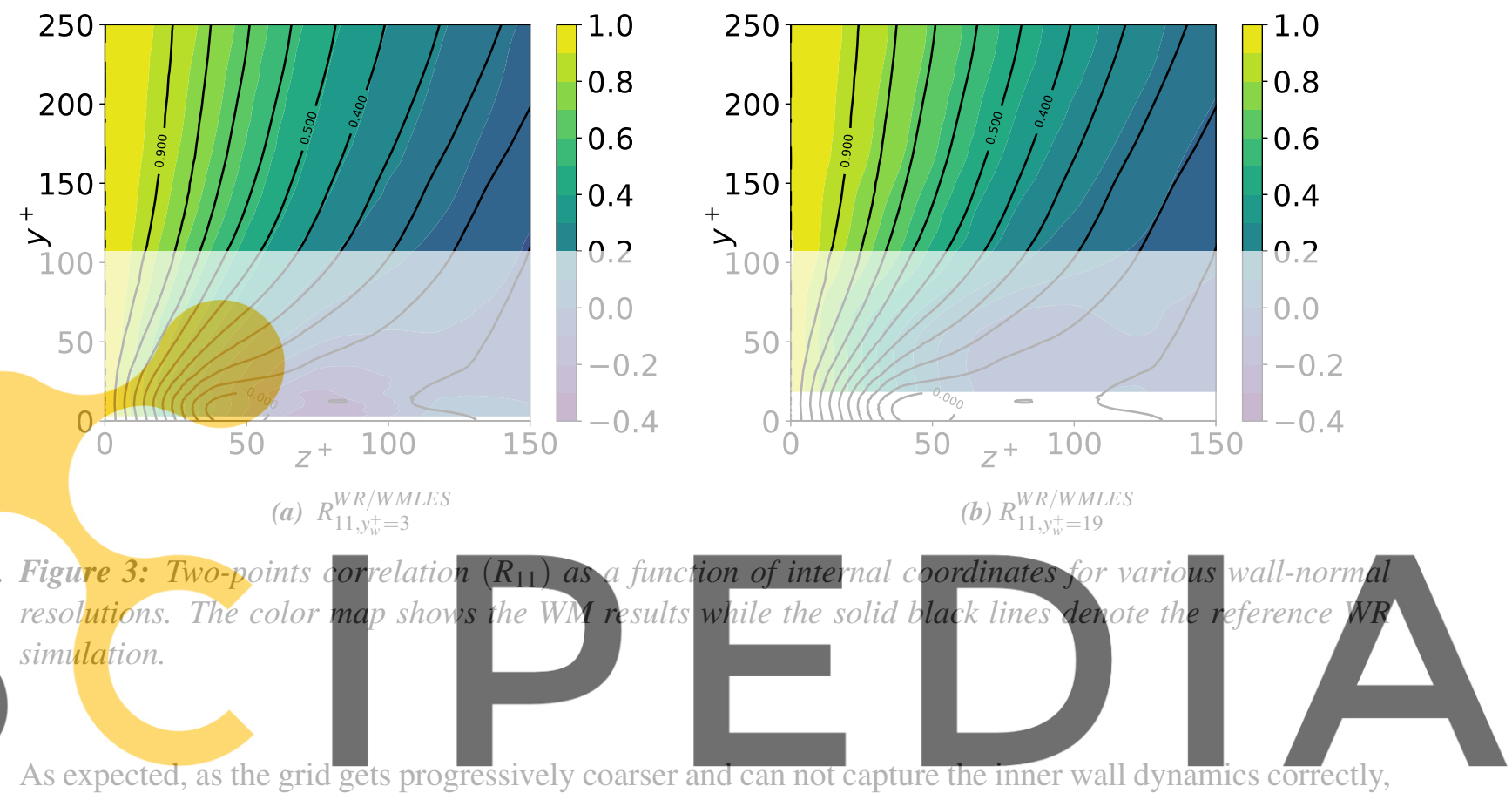

it can still catch the outer dynamics very effectively. Another important consideration is that the external

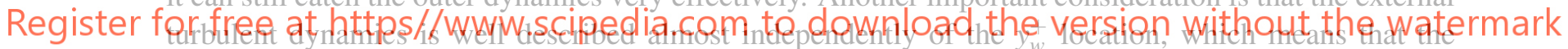

model still provides the correct assessment of the boundary conditions.

\subsection{Spatially-developing compressible turbulent boundary layer}

As a next step, the present unified WR/WMLES is employed to reproduce the dynamics of a spatiallydeveloping compressible turbulent boundary layer over a flat plate. The Mach number of the flow is set equal to $M a_{\infty}=2$ and the inflowing friction Reynolds number is set equal to $R e_{\tau, 0}=\delta_{0} / \delta_{v}=180$. Here $\delta_{0}$ denotes the $99 \%$ inflowing boundary layer thickness while $\delta_{v}=\mu_{w} /\left(\rho_{w} u_{\tau}\right)$ is the viscous length. Wall-normal statistics are collected in correspondence to the location where $R e_{\tau}=250$. The details of the computational setup and boundary conditions can be found looking at Pirozzoli and Bernardini [20]. This type of flow is useful to test the performance of the present unified WR/WMLES also in compressible conditions. As in the previous benchmark, the transition between WR and WM approaches is monitored through progressively coarser grids, starting from an almost WR condition. Thus, the pre-computed $y_{w}^{+}$location is set in the sample $y_{w}^{+}=\{3,19,26\}^{T}$, which represents a coarse WR case, an intermediate WR/WM setups and a mostly WM arrangement. The computations' results are reported in Figure 4 and compared to the DNS data of Pirozzoli and Bernardini [20]. 


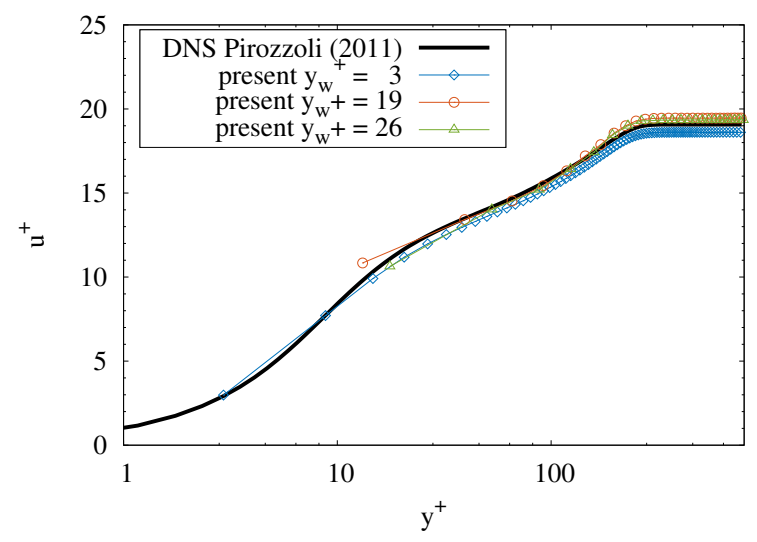

(a) Velocity profile

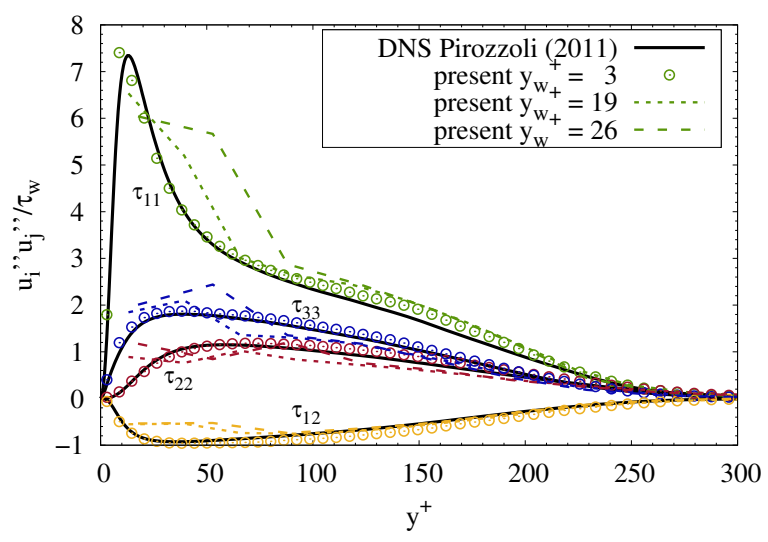

(b) Reynolds stress components

Figure 4: Supersonic spatial-developing turbulent boundary layer with a progressively grid derefinement. Figure $4 a$ and Figure $4 b$ shows the scaled velocity profile $u^{+}=\tilde{u} / u_{\tau}$ and the density scaled Reynolds stress components $\bar{\rho} u_{i}^{\prime \prime} u_{j}^{\prime \prime} / \tau_{w}$ as a function of the $y^{+}$coordinate, respectively. The results correspond to a first cell internal location of $y_{w}^{+}=\{3,19,26\}^{T}$. Present data are compared with the DNS of Pirozzoli and Bernardini [20] at $M a_{\infty}=2$ and $R e_{\tau}=250$.

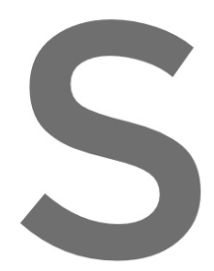

From the obtained results,
for all cases, independentl
profiles disagree with the
it can be seen that away
discrepancy in the fluctuati
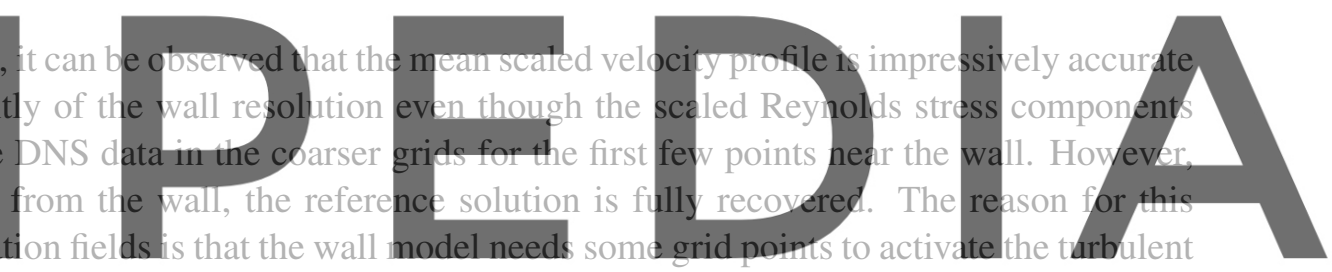

stresses. For this reason, the first points close to the wall are to be considered as a starting region inherent

Register fortlfieenedt.https//www.scipedia.com to download the version without the watermark

\section{CONCLUSIONS}

The paper presents a straightforward numerical strategy able to link the Wall-Resolved (WR) and the Wall-Modelled (WM) Large-Eddy Simulation (LES) approaches in a High-Order Finite-Difference (HOFD) framework. The proposed unified WR/WMLES method locally modifies the diffusive flow properties at the wall location accounting for the SubGrid Scale (SGS) contributions which arise in near-wall lowresolved setups. Moreover, preserving both the no-slip/no-penetration conditions for the velocity and the isothermal/adiabatic conditions for the temperature fields, the proposed numerical strategy falls back into a standard WR case if the grid places enough points in the near-wall regions. This fact implies that the user can save a considerable amount of pre-processing time that is usually taken to treat near-wall regions while getting the most out of its available computational resources.

The numerical technique is applied to nearly-incompressible and compressible turbulent bounded flows configurations proving an excellent accuracy in predicting mean velocity profiles, Reynolds stresses components and two-points spatial correlation in the spanwise direction. In particular, the unified WR/WMLES if found able to accurately head the turbulence dynamics in the outer layer almost in- 
dependently of the near-wall resolution.

Future perspectives of present work will be to implement the unified WR/WMLES method with an Immersed Boundary Method (IBM), (see, e.g., De Vanna et al. [11]) to perform Large-Eddy-Simulations of turbulent flows in complex geometries by using HOFD methods.

\section{References}

[1] Filippo Avanzi, Francesco De Vanna, Yin Ruan, and Ernesto Benini. Design-Assisted of Pitching Aerofoils through Enhanced Identification of Coherent Flow Structures. Designs, pages 1-25, 2021.

[2] Jagadeesh Movva, Dimitrios Papadogiannis, and Stéphane Hiernaux. Assessment of wall modelling for large eddy simulations of turbomachinery. In Turbo Expo: Power for Land, Sea, and Air, volume 51012, page V02CT42A022. American Society of Mechanical Engineers, 2018.

[3] Eran Arad, Neta Yokev, Haim E Brod, and Dan Michaels. From supersonic combustion to thermal choking: Numerical and experimental analysis of a scramjet engine. In AIAA AVIATION 2020 FORUM, page 2966, 2020.

[4] Mattias Liefvendahl, Mattias Johansson, et al. Wall-modeled les for ship hydrodynamics in model scale. Journal of Ship Research, pages 1-14, 2020.

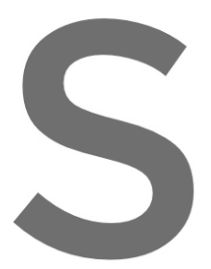

[5] Dean R Chapman. Computational aerodynamics development and outlook. AIAA journal, 17(12): $1293-1313,1979$.

[6] Haecheon Choi and estimates revisited.

[7] Sanjeeb T. Bose Turrbulent Flows.
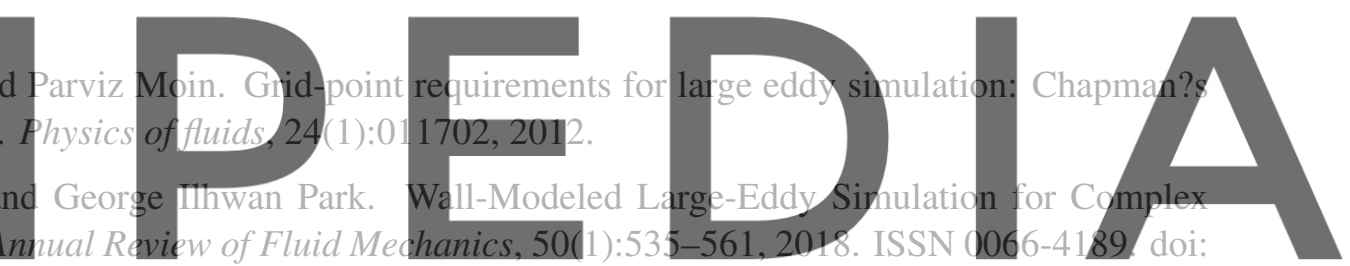

10.1146/annurev-fluid-122316-045241.

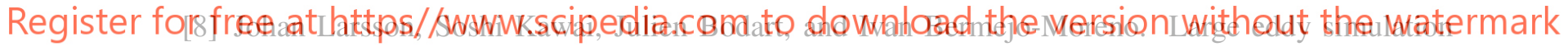
with modeled wall-stress: recent progress and future directions. Mechanical Engineering Reviews, 2016. doi: 10.1299/mer.15-00418.

[9] Sanjeeb T. Bose and George Ilhwan Park. Wall-Modeled Large-Eddy Simulation for Complex Turbulent Flows. Annual Review of Fluid Mechanics, 50(1):535-561, 2018. ISSN 0066-4189. doi: 10.1146/annurev-fluid-122316-045241.

[10] Xiang I.A. Yang and Yu Lv. A semi-locally scaled eddy viscosity formulation for LES wall models and flows at high speeds. Theoretical and Computational Fluid Dynamics, 32(5):617-627, 2018. ISSN 14322250. doi: 10.1007/s00162-018-0471-3.

[11] Francesco De Vanna, Francesco Picano, and Ernesto Benini. A sharp-interface immersed boundary method for moving objects in compressible viscous flows. Computers and Fluids, 201:104415, 2020. ISSN 0045-7930. doi: 10.1016/j.compfluid.2019.104415. URL https://doi.org/10. 1016/j.compfluid.2019.104415.

[12] F. De Vanna, F. Picano, and E. Benini. An Immersed Boundary Method for Moving Objects in Compressible Flows. In In: García-Villalba M., Kuerten H., Salvetti M. (eds) Direct and Large 
Eddy Simulation XII. DLES 2019. ERCOFTAC Series, vol 27. Springer, Cham., volume 27, pages 291-296. 2020. doi: https://doi.org/10.1007/978-3-030-42822-8_38.

[13] Francesco De Vanna, Francesco Picano, and Ernesto Benini. Large-Eddy-Simulations of the unsteady behaviour of a Mach 5 hypersonic intake. (January):1-10, 2021. doi: 10.2514/6.2021-0858.

[14] F. Nicoud and F. Ducros. Subgrid-scale stress modelling based on the square of the velocity gradient tensor. Flow, Turbulence and Combustion, 1999. ISSN 13866184. doi: 10.1023/A:1009995426001.

[15] Francesco De Vanna, Alberto Benato, Francesco Picano, and Ernesto Benini. High order conservative formulation of viscous terms for variable viscosity flows. Acta Mechanica, 2021.

[16] Soshi Kawai and Johan Larsson. Wall-modeling in large eddy simulation: Length scales, grid resolution, and accuracy. Physics of Fluids, 24(1):015105, 2012.

[17] H. Reichardt. Vollständige Darstellung der turbulenten Geschwindigkeitsverteilung in glatten Leitungen. ZAMM - Journal of Applied Mathematics and Mechanics / Zeitschrift für Angewandte Mathematik und Mechanik, 1951. ISSN 15214001. doi: 10.1002/zamm.19510310704.

[18] Matteo Bernardini, Sergio Pirozzoli, and Paolo Orlandi. Velocity statistics in turbulent channel flow up to $\operatorname{Re} \tau=4000$. pages 171-191, 2014. doi: 10.1017/jfm.2013.674.

[19] A. W. Vreman and J. G.M. Kuerten. Statistics of spatial derivatives of velocity and pressure in turbulent channel flow. Physics of Fluids, 2014. ISSN 10897666. doi: 10.1063/1.4891624.

[20] Sergio Pirozzoli and Matteo Bernardini. Turbulence in supersonic boundary layers at moderate
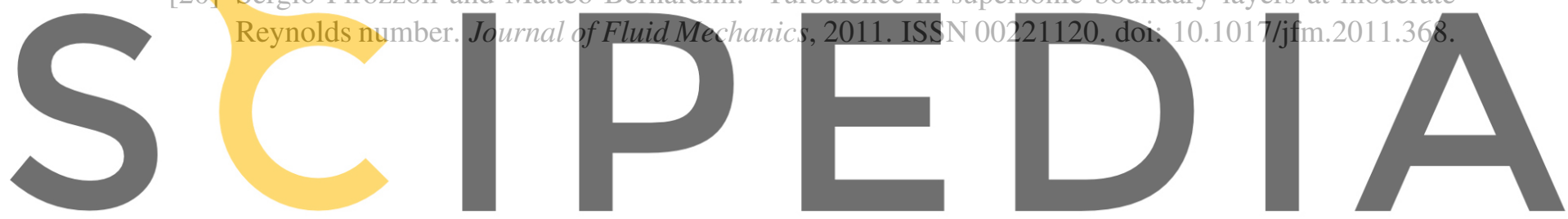

Register for free at https//www.scipedia.com to download the version without the watermark 